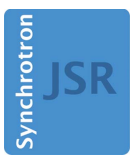

JOURNAL OF SYNCHROTRON RADIATION

ISSN 1600-5775

Received 12 July 2018

Accepted 28 October 2018

Edited by P. A. Pianetta, SLAC National Accelerator Laboratory, USA

Keywords: scintillators; X-ray imaging; thermal behaviour.

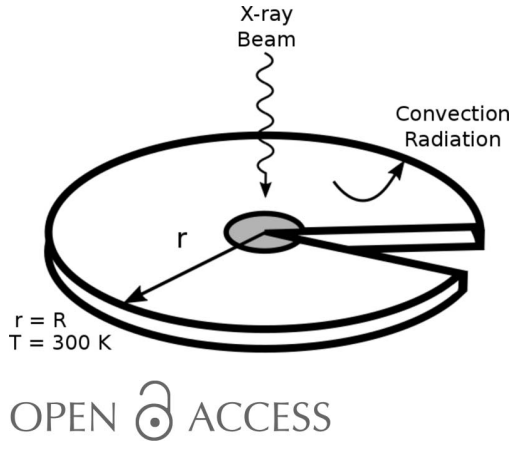

\section{Thermal behavior of single-crystal scintillators for high-speed X-ray imaging}

\author{
Alan Kastengren* \\ Advanced Photon Source, Argonne National Laboratory, 9700 South Cass Avenue, Argonne, IL 60439, USA. \\ *Correspondence e-mail: akastengren@anl.gov
}

Indirect detection of X-rays using single-crystal scintillators is a common approach for high-resolution X-ray imaging. With the high X-ray flux available from synchrotron sources and recent advances in high-speed visible-light cameras, these measurements are increasingly used to obtain time-resolved images of dynamic phenomena. The X-ray flux on the scintillator must, in many cases, be limited to avoid thermal damage and failure of the scintillator, which in turn limits the obtainable light levels from the scintillator. In this study, a transient one-dimensional numerical simulation of the temperature and stresses within three common scintillator crystals (YAG, LuAG and LSO) used for highspeed X-ray imaging is presented. Various conditions of thermal loading and convective cooling are also presented.

\section{Introduction}

A common method for high-resolution X-ray imaging at synchrotrons is indirect imaging with single-crystal scintillators (Bonse \& Busch, 1996; Koch et al., 1998). The X-ray beam illuminates the scintillator, which is in turn imaged with visible-light microscopy. This method can provide resolutions below $1 \mu \mathrm{m}$, which is better than existing detectors which detect X-rays directly or use phosphor powders (Martin \& Koch, 2006). Scintillators are also highly resistant to radiation damage, and this method takes advantage of easily available visible-light microscopy hardware.

A particularly compelling application of X-ray imaging is to study dynamic phenomena, including applications as diverse as the fluid dynamics of fuel injection and sprays (Moon et al., 2014; Halls et al., 2017), mechanical motions inside devices (Baimpas et al., 2013), the flow of blood in blood vessels (Jamison et al., 2012), shock-wave propagation (Olbinado et $a l ., 2017$ ) and the dynamics of metallic foam formation (Rack et al., 2009). To study highly dynamic phenomena, where fast framing rates and short exposure times are essential, a high visible light output from the scintillator is essential, necessitating an intense X-ray beam. White beam, either from a bending magnet or an undulator, is typically used to provide the high X-ray flux needed for such experiments. However, only a small fraction of the X-ray energy absorbed by the scintillator is emitted as visible light, resulting in significant heating of the scintillator. As this heating occurs in a thin crystal of modest dimensions, significant increases in temperature can be expected.

Heating of the scintillator material can have detrimental effects. Anecdotally, it is known that excessive X-ray illumination can lead to cracking of the scintillators, which are typically single crystals and hence quite brittle. Moreover, 
scintillator light output is dependent on temperature (Yanagida et al., 2013). As such, if significant temperature gradients exist in scintillators, the resulting image will not faithfully reflect the distribution of X-ray intensity in the sample, distorting the resulting images. There appears to be little, if any, work in the literature quantifying the heating experienced by scintillators in high-intensity X-ray beams, nor analysis of the resulting stresses in the scintillators.

In the current work, one-dimensional transient thermal simulations are conducted on three commonly used singlecrystal scintillator materials: YAG, LuAG and LSO. Simulations have been performed for both filtered and unfiltered white-beam illumination for a range of scintillator thicknesses and convective heat-transfer coefficients. In addition, the thermal stresses caused by the heating of the scintillator have been calculated and compared with the ultimate stress of the scintillator materials.

\section{Methods}

To simulate the temperatures and stresses in scintillator crystals during illumination from a synchrotron X-ray beam, transient axisymmetric one-dimensional numerical simulations have been undertaken. As such, temperature is a function of radial position $r$ and time $t$. For these simulations, a finite volume approach has been taken. A schematic of the simulation setup is given in Fig. 1. The heat transfer and temperature distributions are assumed to be axisymmetric. The temperature of the outer boundary of the scintillator $(r=R)$ is fixed, as might be expected for a scintillator mounted onto a highly conductive metal holder. Each element of the discretized domain experiences radial heat conduction, convective heat transfer from both free faces to the surroundings and radiative heat transfer from both free faces. An explicit numerical method was used to step through time, using a predictor-corrector approach to improve accuracy. Pertinent parameters of the simulation are given in Table 1; although these values are representative of imaging experiments at the 7-BM beamline of the Advanced Photon Source (APS), the dimensions are similar to those used at other synchrotron beamlines.

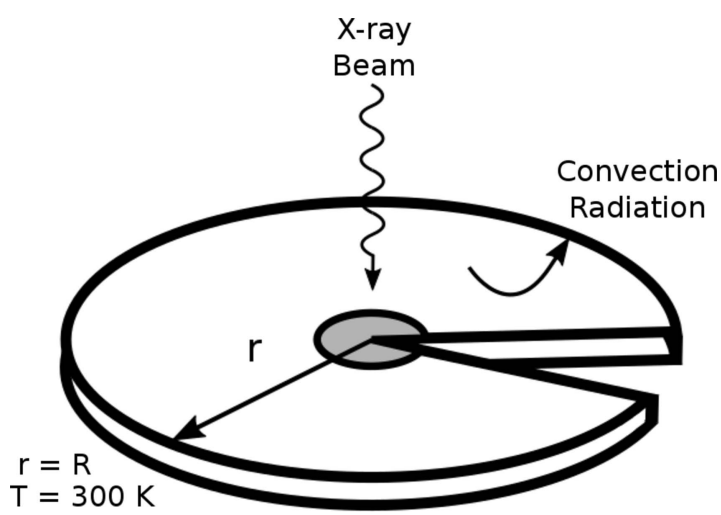

Figure 1

Simulation setup
Table 1

Simulation parameters.

\begin{tabular}{ll}
\hline Scintillator radius $R(\mathrm{~mm})$ & 6 \\
Simulation $\Delta r(\mu \mathrm{m})$ & 25 \\
Scintillator thickness $(\mu \mathrm{m})$ & $5,20,50,100,200$ \\
Illuminated radius $(\mathrm{mm})$ & $0.5,1.0$ \\
Convective heat transfer coefficient $\left(\mathrm{W} \mathrm{m}^{-2} \mathrm{~K}^{-1}\right)$ & $0,1,10,100,300,1000$ \\
Radiative emissivity & 0.5 \\
Outer rim temperature $(\mathrm{K})$ & 300 \\
Surroundings temperature $(\mathrm{K})$ & 300 \\
Simulation time duration $(\mathrm{s})$ & 10 \\
Simulation time step $(\mathrm{s})$ & $5 \times 10^{-6}$ \\
\hline
\end{tabular}

The simulations were initiated with the scintillator at the same temperature as the surroundings $(300 \mathrm{~K})$. A region in the center of the scintillator was then subjected to a heat generation $q_{\text {gen }}$ due to absorption of the X-ray beam. To provide relevant parameters for illumination, the heat loading per unit illuminated area was calculated in XOP (Sánchez del Rio \& Dejus, 2011) for absorption of the beam from an APS bending magnet at a position $35 \mathrm{~m}$ from the source through an aperture $1 \mathrm{~mm} \times 1 \mathrm{~mm}$ in size. Two illumination conditions were simulated for each scintillator material and thickness combination. One is a relatively unfiltered bending-magnet white beam (filtered only with $750 \mu \mathrm{m}$ of beryllium from the beamline windows). This corresponds to illumination used for high-speed imaging. The second condition used illumination additionally filtered with a $250 \mu \mathrm{m}$-thick copper filter. This is a typical configuration used for hard X-ray tomography of dense samples at the APS 7-BM beamline.

Several validation steps were undertaken to ensure the accuracy of the numerical scheme. The results of a simulation without convection or radiation matched the temperature distribution calculated from an analytical solution to the heatconduction equations. For a scintillator with an initial temperature higher than the surroundings, the rate of cooling with radiative and conductive heat transfer but no conduction matched analytical calculations. Tests of convergence in terms of spatial and temporal discretization were also performed and the simulations were found to be well converged with the discretization used. It should be noted that the time step was limited by the stability of the explicit scheme used, not the accuracy of the solution.

Three scintillator materials were simulated in this work. The first is YAG, which has been widely used in X-ray imaging applications for many years. YAG is hard, stable and has a relatively short decay time (Rutherford et al., 2016), which is essential for high-speed imaging, though it has a relatively low effective $Z$, limiting its ability to absorb high-energy X-rays. The second material is LuAG, which shares many properties with YAG, but with a higher effective $Z$. The decay time for LuAG is relatively long, which limits its use for high-speed applications (Rutherford et al., 2016). The final material is LSO. Both LSO and LYSO are fast, high- $Z$ scintillators with high light output, making them attractive for high-speed imaging applications. While LYSO arguably sees more use for imaging applications, far more of the physical property data required for these simulations were available in the literature 
for LSO than LYSO, which is chemically similar to LYSO. All of these materials are doped with cerium for use as scintillators; this doping was ignored for this work, given the paucity of quantitative data on the cerium loading of the different crystals and the potential for variations between scintillator suppliers.

Several assumptions have been made in this analysis. This analysis has ignored the energy emitted by the scintillator as light. This tends to be a relatively small fraction of the total energy input from the X-ray beam, and the literature regarding absolute light output of scintillators tends to be somewhat inconsistent. This analysis also ignores temperature and stress variations through the thickness of the scintillator. While this should be a good assumption for thin scintillators, it may break down for thicker scintillators, particularly under unfiltered white-beam illumination. Fig. 2 shows the energy deposition versus depth in the scintillator for YAG with both unfiltered and filtered illumination based on XOP simulations. The surface illuminated by the beam experiences the greatest degree of heating. The heating rapidly diminishes for unfiltered illumination but diminishes much more slowly for filtered illumination.

The properties of the scintillators are also assumed to be constant with temperature, which will not be correct for scintillators experiencing large heating effects. The scintillators are also assumed to be isotropic materials. This is not true for LSO in particular, but greatly eases the analysis, as it allows the problem to be treated as a $1 \mathrm{D}$ transient heat transfer problem, rather than 2D. It should also be noted that the fixed temperature at the rim of the scintillator may not hold in practice under intense illumination, depending on the thermal conductivity of the holder and the quality of the thermal contact between the scintillator and holder.

Once the temperature distribution of the scintillator as a function of time has been found, an analysis of the thermal stresses of the scintillator can also be performed. Again, the scintillator properties are assumed to be both isotropic and constant with temperature. It is further assumed that the scintillator undergoes plane stress and does not buckle (i.e. the

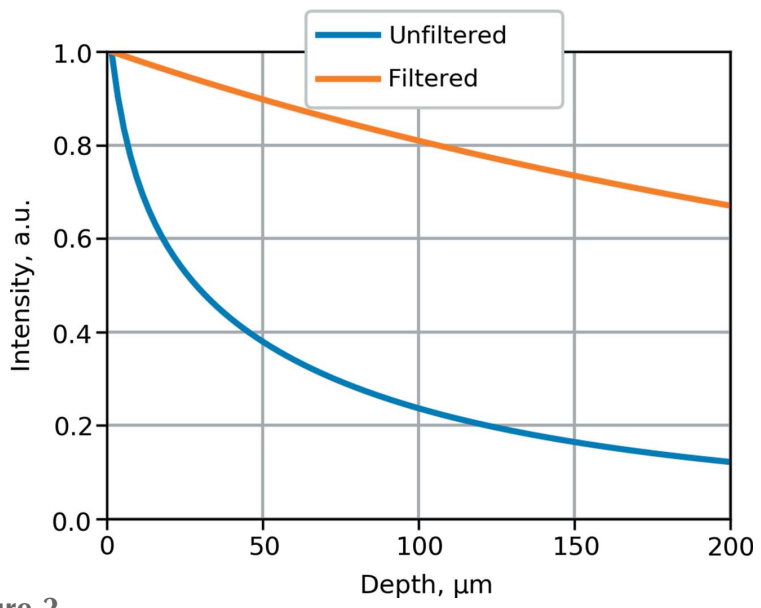

Figure 2

Energy deposition versus depth in the scintillator for YAG under both unfiltered and filtered WB illumination. scintillator remains flat). For an axisymmetric sample undergoing thermal stress, the radial displacement $u$ of the material as a function of $r$ is given by (Bickford, 1998)

$$
u(r)=\frac{1}{r} \int_{0}^{r}(1+v) \alpha b T(b) \mathrm{d} b+C r=u_{\mathrm{T}}(r)+C r .
$$

In this equation, $\alpha$ is the linear thermal expansion coefficient, $v$ is Poisson's ratio, $T$ is the temperature difference between the scintillator at radius $r$ and the surroundings and $C$ is a constant that can be solved by assuming zero radial stress at the edge of the scintillator. The term $u_{\mathrm{T}}$ is used to collect all terms explicitly dependent on the scintillator temperature distribution. This can be solved for the radial and circumferential stresses ( $\sigma_{r}$ and $\sigma_{\theta}$, respectively), using $E$ as the elastic modulus of the scintillator as

$$
\begin{gathered}
\sigma_{r}=\frac{E}{1-v^{2}}\left(u^{\prime}+v \frac{u}{r}\right)-\frac{E \alpha T}{1-v}, \\
\sigma_{\theta}=\frac{E}{1-v^{2}}\left(\frac{u}{r}+v u^{\prime}\right)-\frac{E \alpha T}{1-v}, \\
C=-\frac{1}{1+v}\left[u_{\mathrm{T}}^{\prime}(R)+v \frac{u_{\mathrm{T}}(R)}{R}\right] .
\end{gathered}
$$

Several material properties are needed for the scintillators to complete the thermal simulation. These include thermal conductivity, density and specific heat. To perform the stress analysis of the scintillators, one must also obtain the linear thermal expansion coefficient, elastic modulus, Poisson's ratio and ultimate stress (to judge for failure). The various properties of the scintillators are given in Tables 2-7. It should be noted that for the most part these properties are for undoped parent scintillator materials (finding relevant properties in the literature on the doped crystals was quite challenging). The values for some of the mechanical properties have been assumed based on similar crystals due to the challenges of finding reliable mechanical property data for certain crystals. Poisson's ratio for all crystals is assumed to be 0.25 , which matches the values found in the literature for YAG (Monteseguro et al., 2015). Given the potential variability in mechanical properties depending on crystal growth and processing between suppliers, the aforementioned assumptions seem unlikely to materially add to the error in this analysis.

\section{Results}

Plots of the radial temperature distribution versus time with unfiltered white-beam illumination for the three scintillator materials are given in Fig. 3 for a scintillator thickness of $100 \mu \mathrm{m}$ and an illuminated region $0.5 \mathrm{~mm}$ in radius. For these data, no convective cooling was permitted; these data represent the behavior of scintillators in a vacuum chamber. Several trends are evident. At short times, heating occurs only in the center of the scintillator, corresponding to the region illuminated by the X-ray beam. As time progresses, heat conduction 
Table 2

Thermal conductivity values used in the simulations.

\begin{tabular}{lll}
\hline Crystal & $\begin{array}{l}\text { Thermal conductivity } \\
\left(\mathrm{W} \mathrm{m}^{-1} \mathrm{~K}^{-1}\right)\end{array}$ & Reference \\
\hline YAG & 12.9 & Kuwano et al. $(2004)$ \\
LuAG & 9.6 & Kuwano et al. $(2004)$ \\
LSO & 3.02 & $\begin{array}{c}\text { Cong } \text { et al. }(2009) \text {; average of values } \\
\text { along different crystal orientations }\end{array}$ \\
\hline
\end{tabular}

Table 3

Specific heat values used in the simulations.

\begin{tabular}{lll}
\hline Crystal & $\begin{array}{l}\text { Specific heat, } \\
\left(\mathrm{J} \mathrm{g}^{-1} \mathrm{~K}^{-1}\right)\end{array}$ & Reference \\
\hline YAG & 0.603 & Kuwano et al. $(2004)$ \\
LuAG & 0.411 & Kuwano et al. $(2004)$ \\
LSO & 0.305 & Cong et al. $(2009)$ \\
\hline
\end{tabular}

Table 4

Density values used in the simulations.

\begin{tabular}{lll}
\hline Crystal & $\begin{array}{l}\text { Density } \\
\left(\mathrm{g} \mathrm{cm}^{-3}\right)\end{array}$ & Reference \\
\hline YAG & 4.53 & Kuwano et al. $(2004)$ \\
LuAG & 6.72 & Kuwano et al. $(2004)$ \\
LSO & 7.394 & Cong et al. $(2009)$ \\
\hline
\end{tabular}

Table 5

Elastic modulus values used in the simulations.

\begin{tabular}{lll}
\hline Crystal & $\begin{array}{l}\text { Elastic modulus } \\
(\mathrm{GPa})\end{array}$ & Reference \\
\hline YAG & 271 & Monteseguro et al. (2015) \\
LuAG & 275 for Yb:LuAG & $\begin{array}{l}\text { Fu } \text { et al. } \text { (2016); value is for Yb:LuAG } \\
\text { Scalise } \text { et al. (2011); value is for LYSO }\end{array}$ \\
LSO & 180 & \\
\hline
\end{tabular}

Table 6

Linear thermal expansion coefficient values used in the simulations.

\begin{tabular}{|c|c|c|}
\hline Crystal & $\begin{array}{l}\text { Thermal expansion } \\
\text { coefficient at } 300 \mathrm{~K}\left(\mathrm{~K}^{-1}\right)\end{array}$ & Reference \\
\hline YAG & $6.1 \times 10^{-6}$ & Aggarwal et al. (2005) \\
\hline $\mathrm{LuAG}$ & $6.1 \times 10^{-6}$ & Aggarwal et al. (2005) \\
\hline LSO & $4 \times 10^{-6}$ & $\begin{array}{l}\text { Cong et al. (2009); average of values } \\
\text { along different crystal orientations }\end{array}$ \\
\hline
\end{tabular}

Table 7

Crystal ultimate strength values from the literature.

\begin{tabular}{lll}
\hline Crystal & $\begin{array}{l}\text { Ultimate tensile } \\
\text { stress (MPa) }\end{array}$ & Reference \\
\hline YAG & 175 & Marion (1985) \\
LSO & 90 & Scalise et al. (2011); value is for LYSO \\
\hline
\end{tabular}

causes warming of the surrounding regions as well. A majority of the heating occurs within the first second of illumination; after 5-10 s of illumination, the temperature distribution can largely be considered steady-state. The sharpest temperature gradients are found near the edge of the illuminated region $(r=0.5 \mathrm{~mm})$. Overall, the temperature gradients are modest, indicating that thermal conduction effectively smooths the temperature gradients. It thus seems likely that the neglect of thermal gradients through the scintillator thickness is well justified.

All scintillators show significant degrees of heating. YAG undergoes the least heating, with significantly greater heating for LuAG. The temperature rise of the LSO crystal is, by comparison, quite remarkable. These trends, which were seen throughout the simulations, were a consequence of two factors. First, LuAG and LSO have significantly greater X-ray absorption than YAG and, as such, absorb significantly more $\mathrm{X}$-ray flux. Second, the thermal diffusivity of YAG is somewhat higher than LuAG, which is in turn far higher than that of

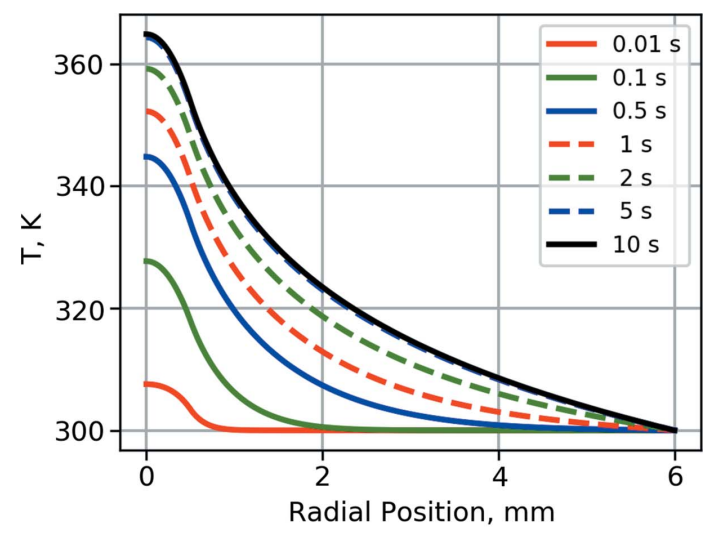

(a)

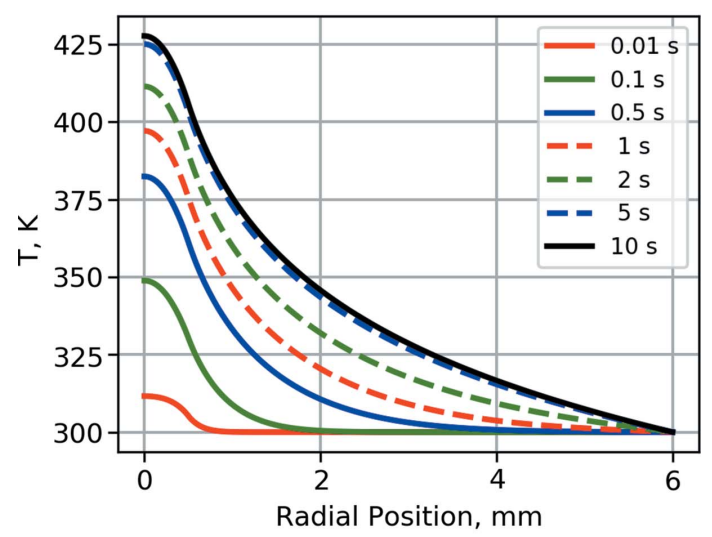

(b)

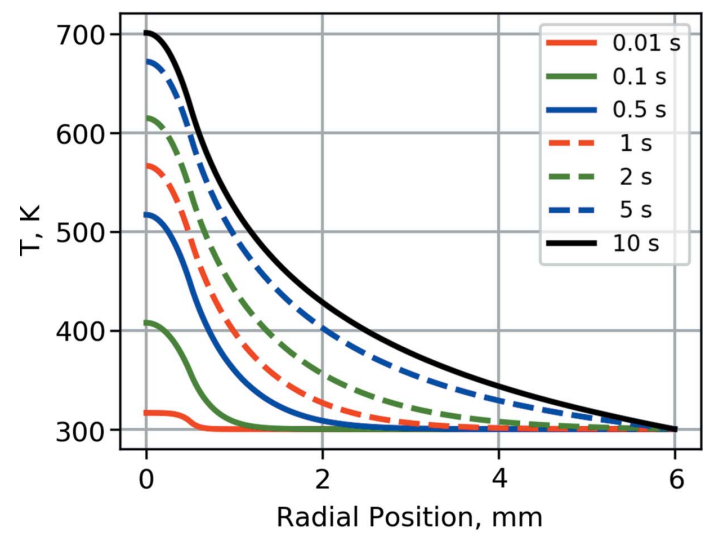

Figure 3

(c)

Radial distribution of scintillator temperature versus time for unfiltered white-beam illumination, $0.5 \mathrm{~mm}$ illuminated radius, $100 \mu \mathrm{m}$ scintillator thickness for (a) YAG, (b) LuAG and (c) LSO. 
LSO. Lower values of thermal diffusivity inhibit the conduction of heat away from the illuminated region and into the scintillator holder, leading to higher temperatures throughout the scintillator. The combination of these two factors leads to the dramatically higher temperatures for LSO compared with YAG and LuAG. It should be noted that these degrees of temperature rise call into question the assumption of constant mechanical and thermal properties used for this analysis.

Fig. 4 shows the temperature at the center of the scintillators as a function of time under white-beam illumination for various scintillator thicknesses. As shown in Fig. 3, the YAG scintillator demonstrates the lowest temperatures, followed by LuAG, with LSO demonstrating the highest temperatures. For all thicknesses, the vast majority of the heating is complete within $5 \mathrm{~s}$ of the onset of illumination. The time period of the thermal transient is weakly dependent on thickness, with thicker scintillators reaching thermal equilibrium more slowly.

The thickness also clearly has a significant impact on the scintillator temperature. Except for LSO, the thinnest scintillators show the highest temperatures. This is tied directly to the absorption of the beam by the scintillator. As Fig. 2 demonstrates, the illuminated surface of the scintillator experiences the greatest X-ray flux, especially for unfiltered white beam. As scintillator thickness increases, attenuation of the beam causes the amount of heat deposition to increase more slowly than the thickness, while the cross-sectional area available for conduction increases linearly with thickness. Thus, the added thermal loading for thicker scintillators is more than compensated for by the greater cross-sectional area available for heat conduction, leading to lower overall temperatures than for thinner scintillators.

The temperature history of the scintillators is quite different when the illumination is filtered. Fig. 5 shows the centerline time history for the scintillators, like in Fig. 4, but with filtered white-beam illumination. The temperatures of the different thicknesses are much lower than for unfiltered beam, which is expected due to the lower heat load. The temperatures for the different thicknesses are all much closer to each other than in Fig. 4. This is again logical; as shown in Fig. 2, the total heat loading will increase nearly linearly with additional thickness, nearly balancing the effect of greater cross sectional area for conduction. The thinner scintillators still show a slightly faster approach to thermal equilibrium than thicker scintillators. The highest temperatures are no longer seen in the thinnest scintillators, which is probably the result of radiative losses at the surface of the scintillator, which play a greater role for the thinner scintillators. Despite the filtering, however, the LSO scintillator still shows a significant temperature rise due to illumination by the X-ray beam.

One of the major concerns with the heating of scintillators is fracture of the scintillators due to thermal stresses. Fig. 6 shows the thermal stress as a function of $r$ for the three scintillators with and without filtering of the illumination $10 \mathrm{~s}$ after the start of illumination (i.e. virtually steady-state). The stress distribution is compressive near the center of the scintillator, with tensile stresses near the edge. This is logical, since the center of the scintillator expands due to heating much more

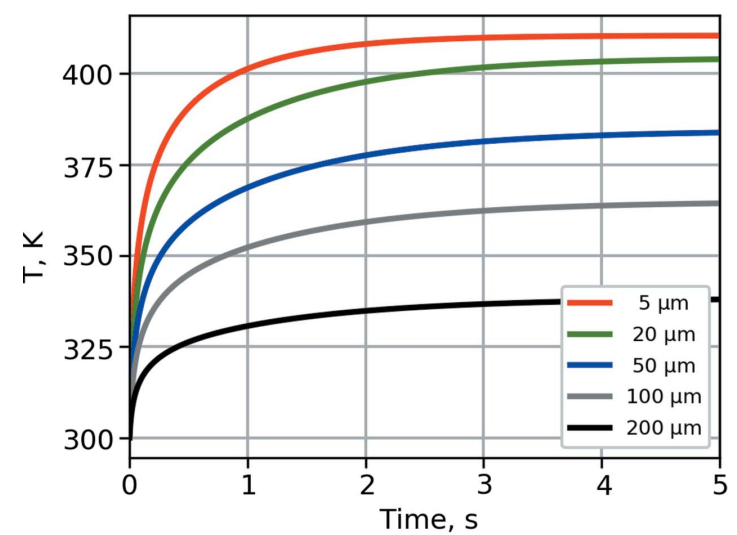

(a)

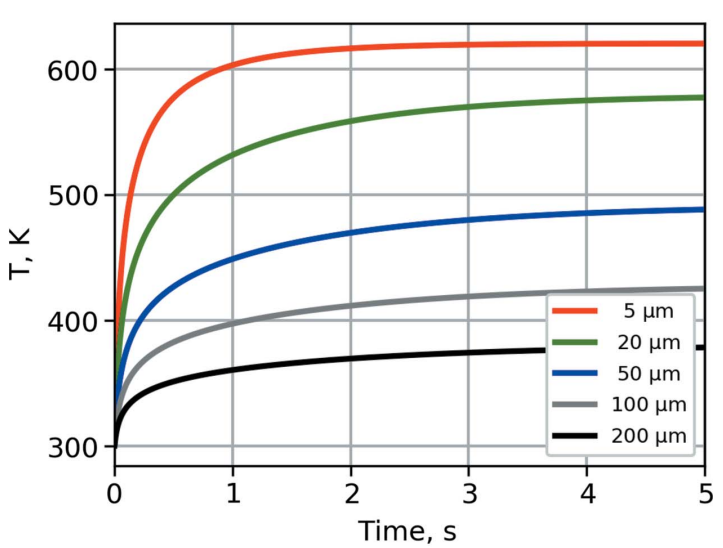

(b)

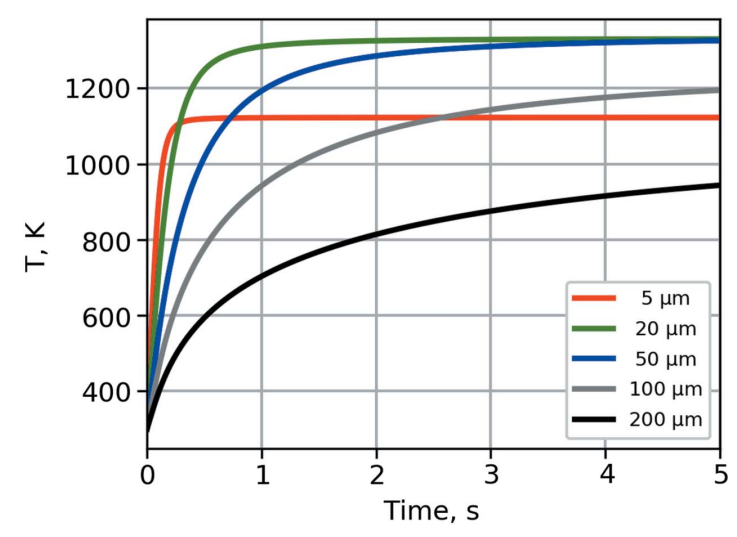

(c)

Figure 4

Centerline scintillator temperature versus time for various scintillator thicknesses. Unfiltered white-beam illumination, $0.5 \mathrm{~mm}$ illuminated radius for (a) YAG, (b) LuAG and (c) LSO.

than the edges. The highest stress magnitudes are consistently found in the center of the scintillator. The trends in the stress magnitude follow the trends in temperature: the highest stresses are found for LSO, the next highest for LuAG and the lowest stresses for YAG.

These results help to explain the anecdotal finding that LSO is more easily damaged by intense illumination than LuAG or YAG. The stress magnitude is much greater in LSO than the other crystals. As shown in Table 7, the ultimate strength of 


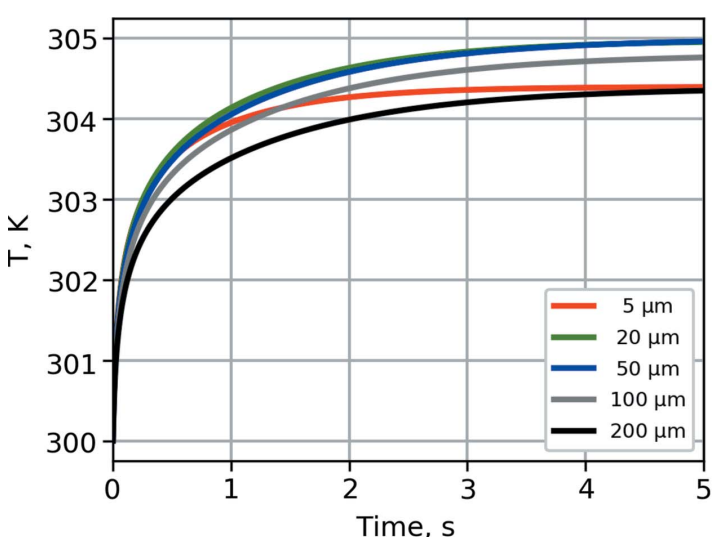

(a)

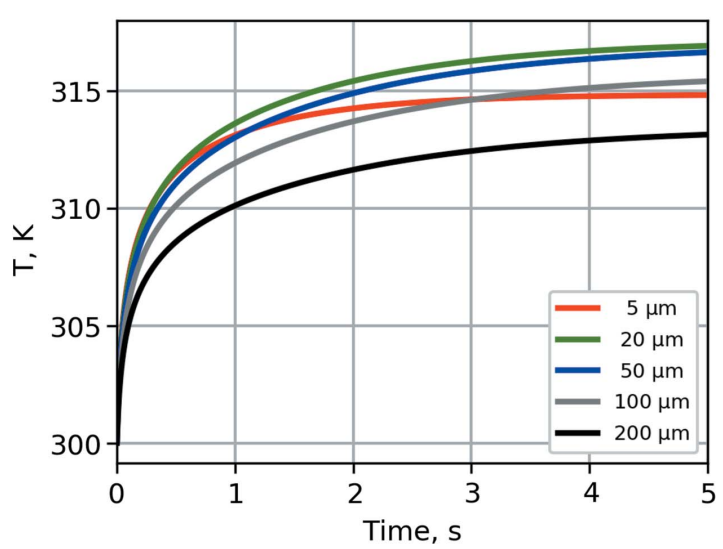

(b)

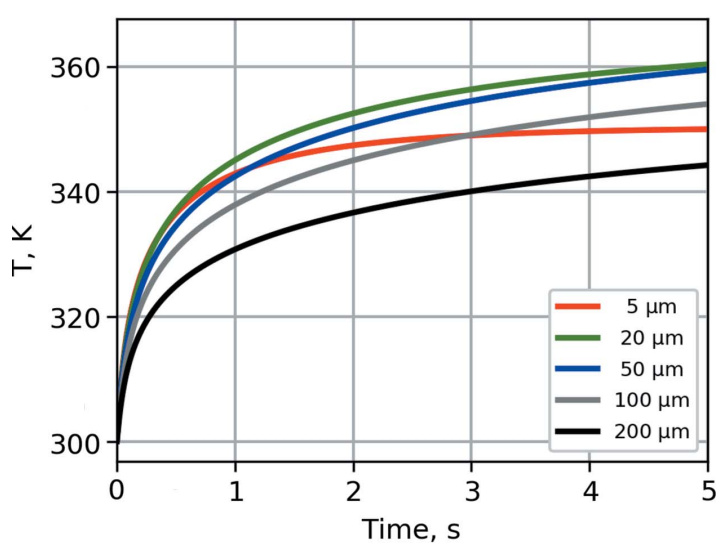

(c)

Figure 5

Centerline scintillator temperature versus time for various scintillator thicknesses. Filtered white-beam illumination, $0.5 \mathrm{~mm}$ illuminated radius for (a) YAG, (b) LuAG and (c) LSO.

LSO is also much lower than YAG. As such, LSO will fracture at a much lower level of illumination than the other crystals. Even with filtered illumination, the compressive stresses at the center of the LSO crystal are a significant fraction of the crystal's ultimate strength. Furthermore, the anisotropic material properties of LSO may add additional stress to the crystal beyond what is shown in this analysis, which assumes isotropic material properties.

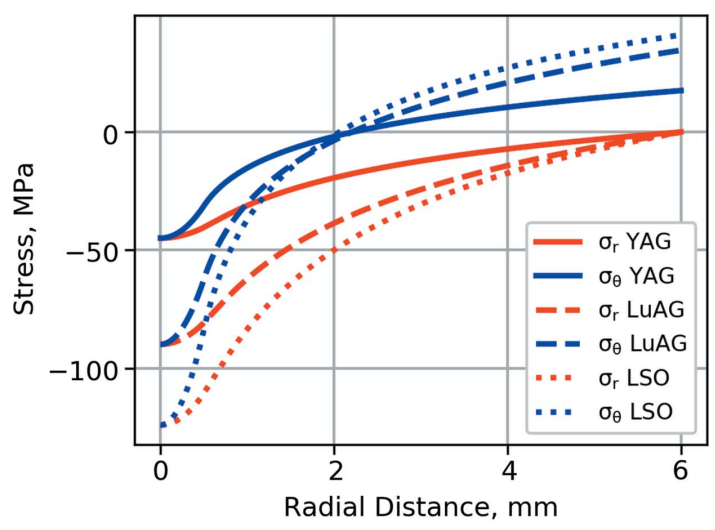

(a)

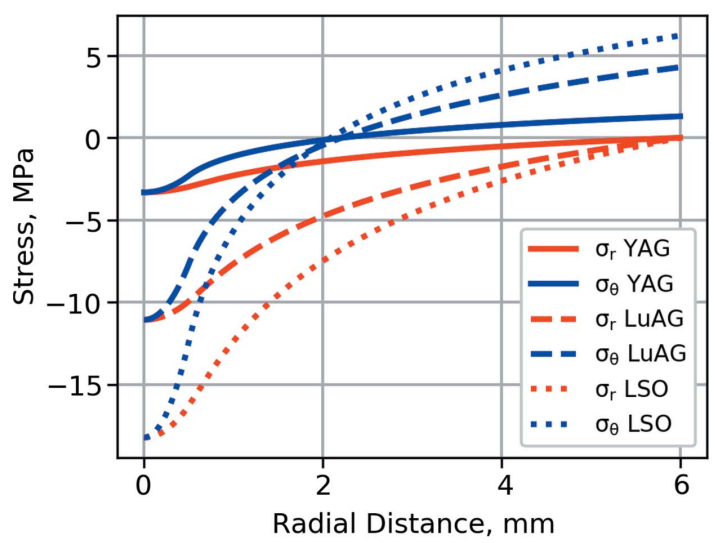

(b)

Figure 6

Steady-state radial and circumferential thermal stress for $100 \mu \mathrm{m}$-thick scintillators under $(a)$ unfiltered and $(b)$ filtered white-beam illumination. No convective heat transfer, $0.5 \mathrm{~mm}$ illuminated radius.

As might be expected, illumination over a larger area leads to higher temperatures and stresses. Fig. 7 shows the centerline temperature history for YAG and LSO; the behavior for LuAG is intermediate between these two examples. The steady-state stress for all three scintillators with illumination over a region $2 \mathrm{~mm}$ in diameter can be seen in Fig. 8, compared with the $1 \mathrm{~mm}$ illumination diameter in Fig. 6. The temperature rise of the scintillator is much higher than for the $1 \mathrm{~mm}$ illumination diameter. The stress fields also become much larger in magnitude. For unfiltered beam, the peak stress magnitude is now a large fraction of the ultimate stress of LuAG. For filtered beam, the peak stress of the LSO crystal is approximately half of the ultimate stress of the crystal. Given the potential errors due to the assumptions underpinning this analysis (isotropic material properties, fixed outer rim temperature), failure of LSO under constant filtered beam illumination is a distinct possibility.

The previous results have all assumed no convective cooling of the scintillators, such as would be found in vacuum operation. In normal use, scintillators are used in air or another gas environment, raising the possibility of either natural or forced convective cooling of the scintillator. Figs. $9(a)-9(c)$ show the time history of the radial temperature distribution with various convective heat-transfer coefficients for a YAG scin- 


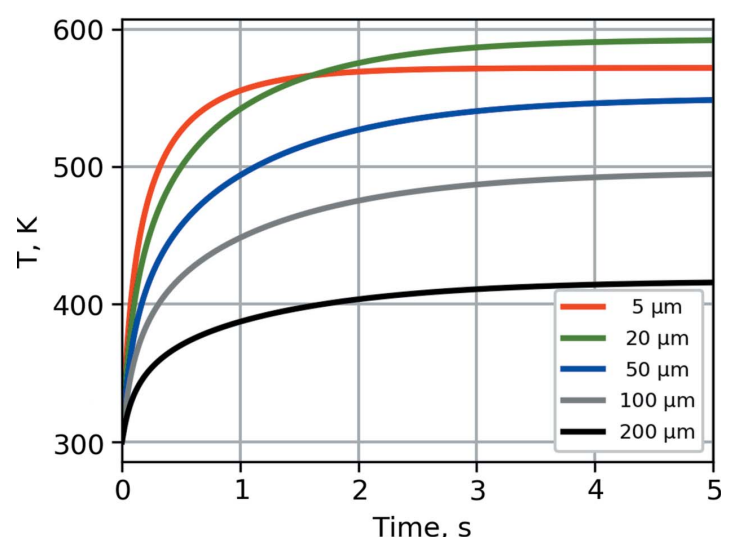

(a)

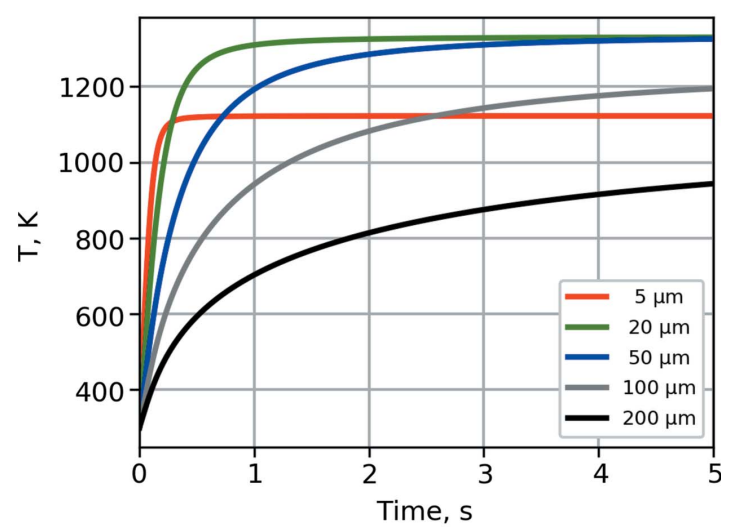

(b)

Figure 7

Centerline scintillator temperature versus time for various scintillator thicknesses. Unfiltered white-beam illumination, $1.0 \mathrm{~mm}$ illuminated radius for $(a)$ YAG and $(b)$ LSO.

tillator, and can be compared with Fig. 3(a), which shows the same case with no convective heat transfer; the trends are similar for other scintillators. The addition of $h=$ $100 \mathrm{~W} \mathrm{~m}^{-2} \mathrm{~K}^{-1}$ convective heat transfer slightly decreases the ultimate temperature of the scintillator. The effects become more significant at $300 \mathrm{~W} \mathrm{~m}^{-2} \mathrm{~K}^{-1}$. Convective heat transfer has three major effects. First, the peak temperature in the scintillator is reduced. Second, the temperature of the unilluminated region of the crystal becomes much closer to the ambient temperature. Third, the time evolution of the temperature distribution becomes much more rapid. The heating of the crystal at short timescales $(<0.1 \mathrm{~s})$ is barely affected by the convective cooling. However, the heating process largely ends by $0.5 \mathrm{~s}$ after the start of illumination.

Fig. 10 shows the time history of the centerline temperature for various YAG scintillator thicknesses for $h=300 \mathrm{~W} \mathrm{~m}^{-2}$ $\mathrm{K}^{-1}$ convective heat-transfer coefficient. When compared with Fig. 4(a), the temperatures are all lower; this effect is quite dramatic for the thinner scintillators. Convection occurs only on the surfaces of the scintillator, and as such is quite effective at cooling thin scintillators, while having a more minor effect for thicker scintillators. This reduction in heating significantly reduces the stresses within the crystals, especially for thin crystals, as shown in Fig. 11.

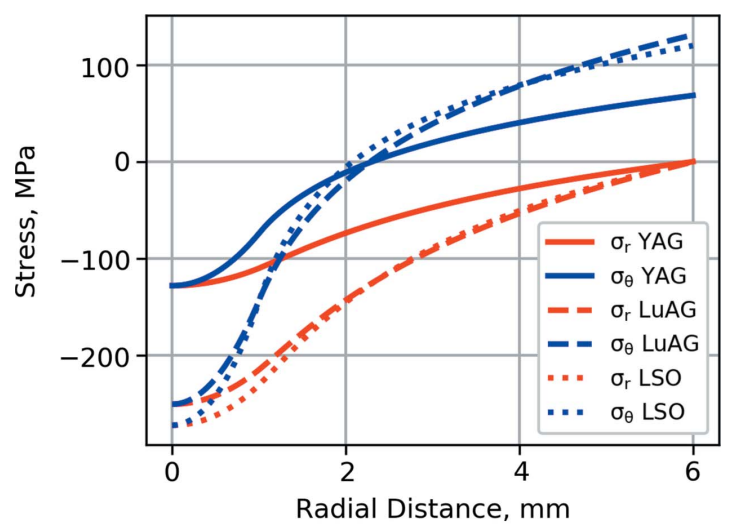

(a)

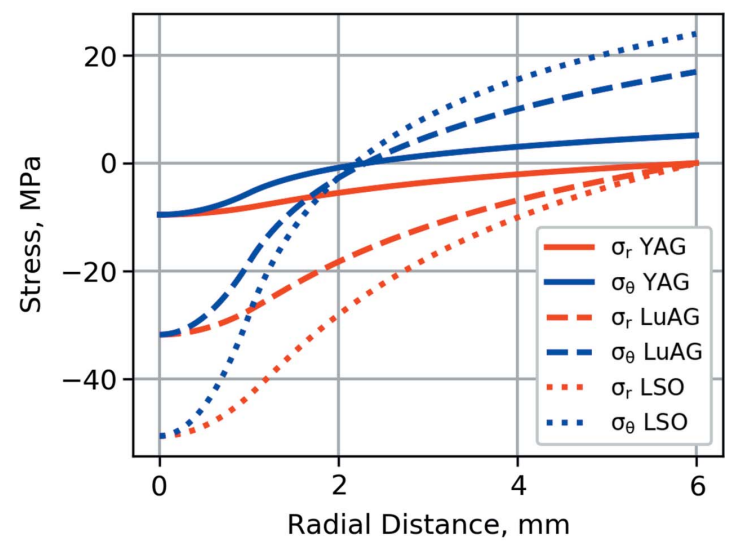

(b)

Figure 8

Steady-state radial and circumferential thermal stress for $100 \mu \mathrm{m}$-thick scintillators under $(a)$ unfiltered and $(b)$ filtered white-beam illumination. No convective heat transfer, $1.0 \mathrm{~mm}$ illuminated radius.

\section{Discussion}

While the previous results show clearly that thermal loading due to intense synchrotron beams can lead to the mechanical failure of scintillators, a further issue is changes in the scintillator light output as a result of temperature changes. For example, if a fast shutter is used to control the illumination of the scintillator, heating of the scintillator can cause temporal drifts in the scintillator light output. If one attempts to image dynamic phenomena, such temporal drifts in light conversion efficiency of the scintillator will make quantitative absorption measurements of dynamic systems difficult.

Relatively little published data exist regarding the sensitivity of scintillator light output to temperature effects. Existing literature data suggest that YAG shows a decrease in light output for a given illumination as temperature increases above room temperature, though LuAG:Pr shows an increase in light output at moderately elevated temperatures, which may also occur for LuAG:Ce (Yanagida et al., 2013). Given the temperature gradients in the scintillators, this should appear as non-uniform light output. An example to demonstrate such effects is shown in Fig. 12, taken from a series of liquid spray images. White beam from an APS bending magnet at the 7-BM beamline was filtered with $750 \mu \mathrm{m}$ beryllium (from 


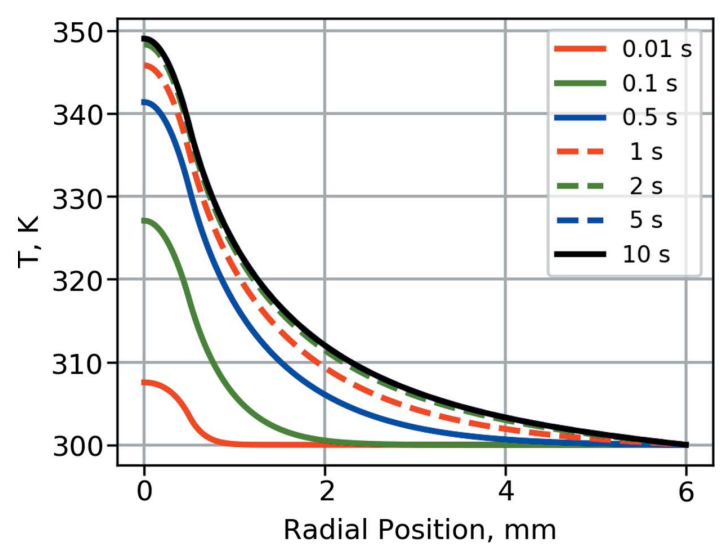

(a)

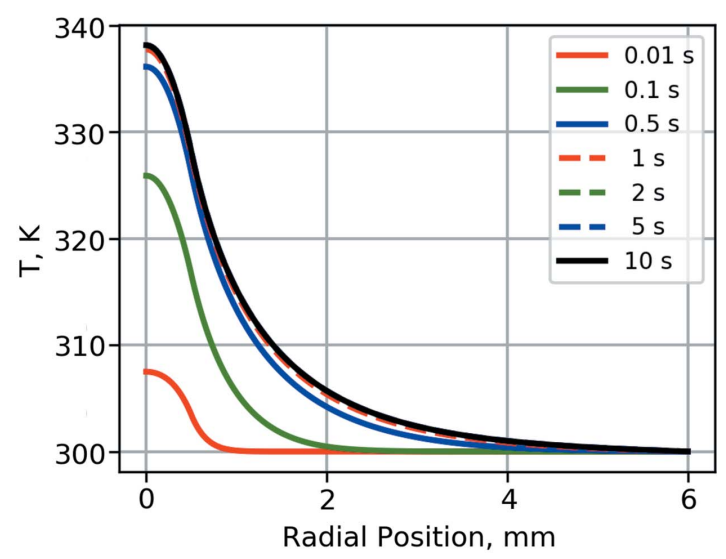

(b)

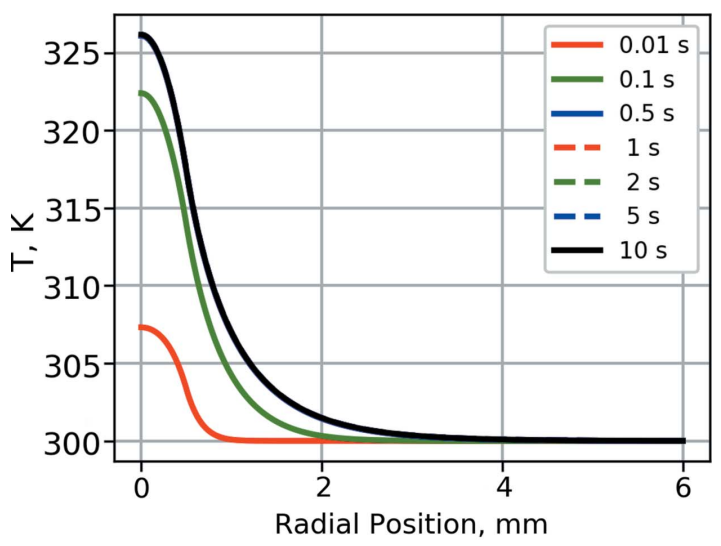

(c)

Figure 9

Centerline scintillator temperature versus time for various convective heat transfer coefficients. Unfiltered white-beam illumination, $0.5 \mathrm{~mm}$ illuminated radius, YAG scintillator, $100 \mu \mathrm{m}$ thickness for $h\left(\mathrm{~W} \mathrm{~m}^{-2} \mathrm{~K}^{-1}\right)=(a) 100,(b) 300$ and $(c) 1000$.

beamline windows), then illuminated a $100 \mu \mathrm{m}$-thick, $12 \mathrm{~mm}$ diameter LuAG:Ce scintillator located $36 \mathrm{~m}$ from the X-ray source. A Photron SA-4 high-speed camera recorded images of the scintillator for a time period of $3 \mathrm{~s}$ after the beamline shutter was opened. As the scintillator was illuminated, the light output in the center of the scintillator first increased slightly, then decreased markedly, as would be expected from the previous results. In this case, the scintillator is clearly

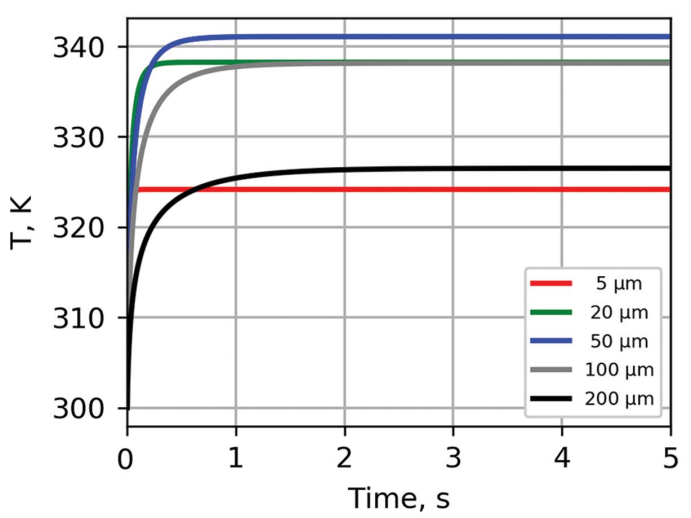

Figure 10

Centerline scintillator temperature versus time for various scintillator thicknesses. Unfiltered white-beam illumination, $0.5 \mathrm{~mm}$ illuminated radius, YAG scintillator and $h=300 \mathrm{~W} \mathrm{~m}^{-2} \mathrm{~K}^{-1}$.

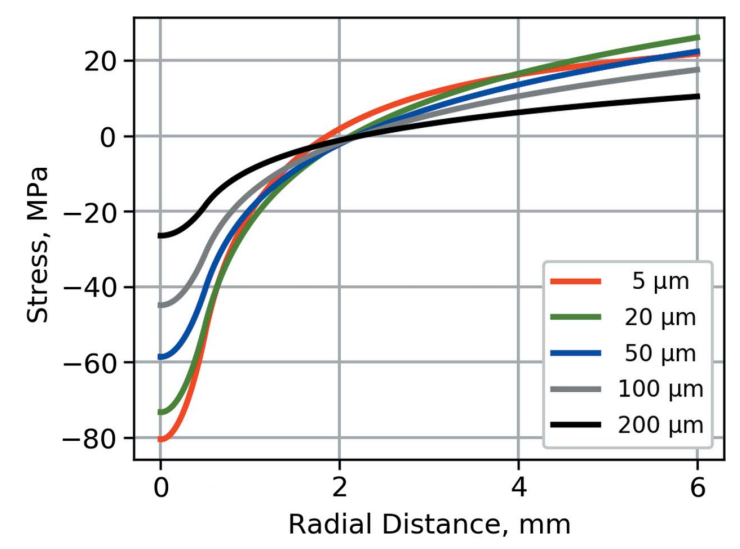

(a)

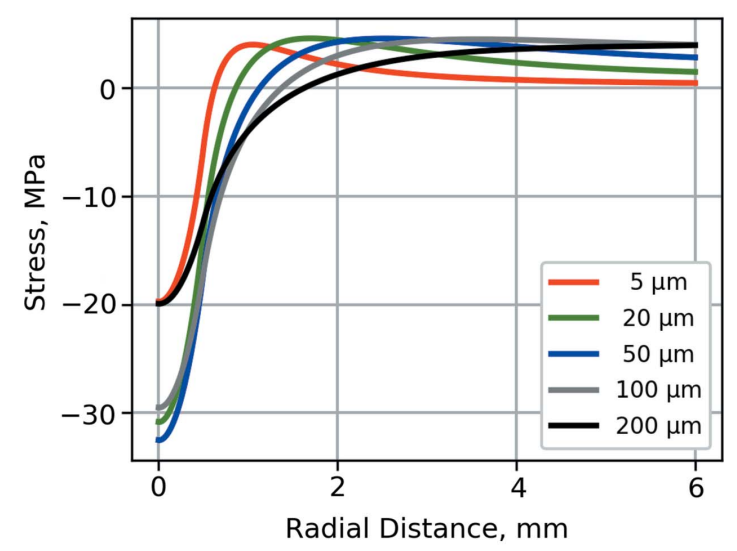

Figure 11

(b)

Circumferential stress for various scintillator thicknesses. Unfiltered white-beam illumination, $0.5 \mathrm{~mm}$ illuminated radius, YAG scintillator for (a) $h=0 \mathrm{~W} \mathrm{~m}^{-2} \mathrm{~K}^{-1}$ and $(b) h=300 \mathrm{~W} \mathrm{~m}^{-2} \mathrm{~K}^{-1}$.

undergoing a great deal of heating in the center of the illuminated area. After further filtering this beam with $500 \mu \mathrm{m}$ of silicon, the beam was allowed to illuminate the scintillator for a longer period of time; the crystal mechanically failed after approximately $10 \mathrm{~s}$ of illumination.

Several strategies exist to mitigate changes in light output caused by heating, though they have drawbacks. Fast shut- 


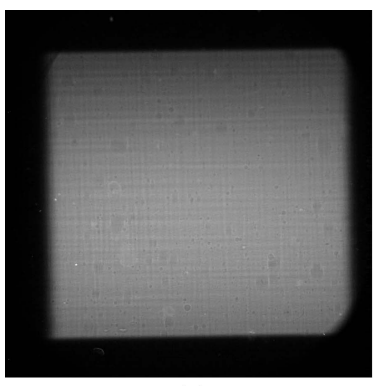

(a)

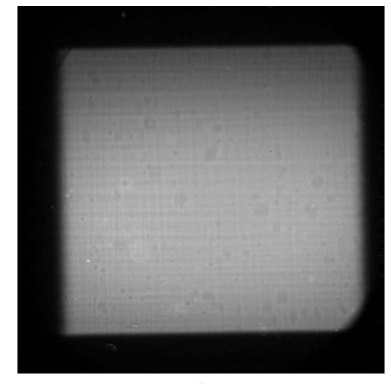

(b)

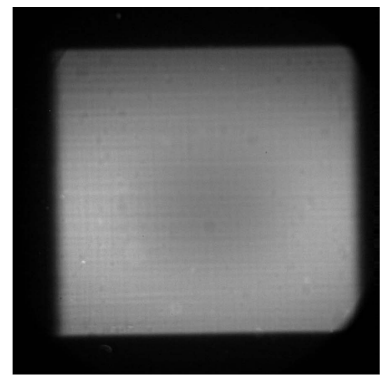

(c)

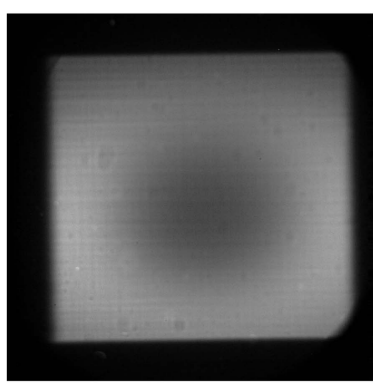

(d)

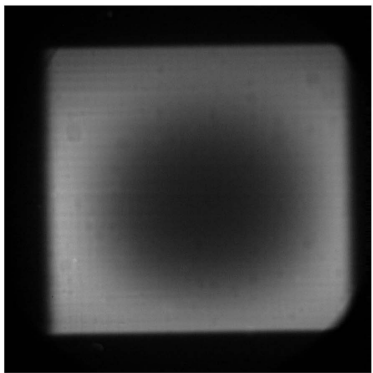

(e)

Figure 12

Images of scintillator output when exposed to an intense X-ray beam. LuAG scintillator, $100 \mu \mathrm{m}$ thickness, 12 mm diameter, located $36 \mathrm{~m}$ from the X-ray source. Illumination from unfiltered APS bending-magnet white beam (beam size $3.15 \mathrm{~mm} \times 3.15 \mathrm{~mm}$ ). Images were recorded after the start of illumination of $(a) 0.1 \mathrm{~s},(b) 1.0 \mathrm{~s},(c) 1.5 \mathrm{~s},(d) 2.0 \mathrm{~s}$ and $(e) 3.0 \mathrm{~s}$.

tering of the X-ray beam can limit thermal effects by limiting the total energy deposited in the scintillator, and has been applied quite successfully at some beamlines, though this limits the time duration over which imaging can be performed. Thicker scintillators suffer less heating and lower thermal stresses, but limit the resolution possible with an imaging system. The use of more thermally stable scintillators can allow for greater illumination of the scintillator without thermal damage. Filtering of the incident beam is also helpful, but changes the signal level and spectrum (for polychromatic beams).

Convective cooling provides an alternative method to combat beam-induced heating. While ineffective for short pulses of X-rays, it can significantly reduce scintillator temperatures for longer exposure imaging, especially for thin scintillators. The convective heat-transfer coefficients simulated in this work are high, but can be reasonably achieved with the impingement of a gas jet on the scintillators (Yan \& Saniei, 1997). Moreover, recent work (Zhou et al., 2018) suggests that purging inert gas around scintillators can prevent soiling of the scintillators. By directing this gas flow appropriately, it could be used for both maintaining scintillator cleanliness and providing active cooling.

It is clear from the above results that heating effects are especially severe for thin scintillators unless steps are taken to reduce thermal loading. This is unfortunate, since thin scintillators are preferred for high-resolution imaging due to the limited depth of field of high-magnification microscopes. Thin scintillators cannot absorb as much of a high-energy X-ray beam as thick scintillators, so a potential avenue to achieve adequate light output for time-resolved measurements at high spatial resolution is to use a more intense X-ray beam. These simulations show that such approaches must be taken with great care to avoid thermal damage to the scintillator.

\section{Conclusions}

Finite-volume simulations of the thermal response of three common X-ray scintillator materials to both filtered and unfiltered white-beam illumination have been performed. The simulations included the influence of thermal conduction, convection and radiation. Across all simulations, YAG experienced the lowest degree of heating, followed by LuAG, with LSO experiencing by far the highest degree of heating; this is directly tied to the absorption and heat transfer properties of the scintillator materials. For unfiltered beam, thinner scintillators experienced the greatest degree of heating, while this effect is greatly reduced with filtering of the incident beam. The degree of heating is such that stress levels exceeding the ultimate stress of the scintillator materials can be induced. The introduction of convective cooling can reduce the heating seen in the scintillators, especially for thin scintillators, though high levels of convection are needed to achieve such cooling.

\section{Acknowledgements}

The author would like to thank Katarzyna Matusik from the Argonne X-ray Science Division for discussions of this work and assistance in obtaining the images in Fig. 12. 


\section{Funding information}

The measurements in this work were conducted at the 7-BM beamline of the Advanced Photon Source and were supported by the US Department of Energy (DOE), Office of Science, Office of Basic Energy Sciences (Contract No. DE-AC0206CH11357).

\section{References}

Aggarwal, R. L., Ripin, D. J., Ochoa, J. R. \& Fan, T. Y. (2005). J. Appl. Phys., 98, 103514.

Baimpas, N., Drakopoulos, M., Connolley, T., Song, X., Pandazaras, C. \& Korsunsky, A. M. (2013). J. Synchrotron Rad. 20, 316-323.

Bickford, W. (1998). Advanced Mechanics of Materials. Menlo Park, CA: Longman.

Bonse, U. \& Busch, F. (1996). Prog. Biophys. Mol. Biol., 65, 133-169.

Cong, H., Zhang, H., Wang, J., Yu, W., Fan, J., Cheng, X., Sun, S., Zhang, J., Lu, Q., Jiang, C. \& Boughton, R. I. (2009). J. Appl. Cryst. 42, 284-294.

Fu, Y., Li, J., Wang, C., Xie, T., Li, W., Wu, L. \& Pan, Y. (2016). J. Alloy. Compd, 664, 595-601.

Halls, B. R., Radke, C. D., Reuter, B. J., Kastengren, A. L., Gord, J. R. \& Meyer, T. R. (2017). Opt. Express, 25, 1605.

Jamison, R. A., Siu, K. K. W., Dubsky, S., Armitage, J. A. \& Fouras, A. (2012). J. Synchrotron Rad. 19, 1050-1055.
Koch, A., Raven, C., Spanne, P. \& Snigirev, A. (1998). J. Opt. Soc. Am. A, 15, 1940.

Kuwano, Y., Sudo, K., Ishizawa, N. \& Yamada, T. (2004). J. Cryst. Growth, 260, 159-165.

Marion, J. (1985). Appl. Phys. Lett. 47, 694-696.

Martin, T. \& Koch, A. (2006). J. Synchrotron Rad. 13, 180-194.

Monteseguro, V., Rodriguez-Hernandez, P. \& Munoz, A. (2015). J. Appl. Phys. 118, 245902.

Moon, S., Gao, Y., Wang, J., Fezzaa, K. \& Tsujimura, T. (2014). Fuel, 133, 299-309.

Olbinado, M. P., Just, X., Gelet, J-.L., Lhuissier, P., Scheel, M., Vagovic, P., Sato, T., Graceffa, R., Schulz, J., Mancuso, A., Morse, J. \& Rack, A. (2017). Opt. Express, 25, 13857-13871.

Rack, A., García-Moreno, F., Baumbach, T. \& Banhart, J. (2009). J. Synchrotron Rad. 16, 432-434.

Rutherford, M. E., Chapman, D. J., White, T. G., Drakopoulos, M., Rack, A. \& Eakins, D. E. (2016). J. Synchrotron Rad. 23, 685693.

Sánchez del Río, M. \& Dejus, R. J. (2011). Proc. SPIE, 8141, 814115.

Scalise, L., Rinaldi, D., Davi, F. \& Paone, N. (2011). Nucl. Instrum. Methods Phys. Res. B, 654, 122-126.

Yanagida, T., Fijimoto, Y., Kurosawa, S., Kamada, K., Takahashi, H., Fukazawa, Y., Nikl, M. \& Chani, V. (2013). Jpn. J. App. Phys. 52, 076401.

Yan, X. \& Saniei, N. (1997). Intl J. Heat Fluid Flow, 18, 591-599.

Zhou, T., Wang, H., Connolley, T., Scott, S., Baker, N. \& Sawhney, K. (2018). J. Synchrotron Rad. 25, 801-807. 